\title{
Effects of Benzalkonium Chloride on Planktonic Growth and Biofilm Formation by Animal Bacterial Pathogens
}

\author{
Azizollah Ebrahimi ${ }^{1}$; Majid Hemati ${ }^{2, *}$; Ziba Shabanpour $^{2}$; Saeed Habibian Dehkordi ${ }^{\text {; }}$; \\ Shahab Bahadoran ${ }^{1}$; Sharareh Lotalian ${ }^{1}$; Shahin Khoibani ${ }^{2}$ \\ ${ }^{1}$ Department of Pathobiology, School of Veterinary Sciences, Shahrekord University, Shahrekord, IR Iran \\ ${ }^{2}$ School of Veterinary Sciences, Shahrekord University, Shahrekord, IR Iran \\ ${ }^{*}$ Corresponding author: Majid Hemati, School of Veterinary Sciences, Shahrekord University, P.O.Box: 115, Postal Code: 88186/34141, IR Iran. Tel: +98-9352611155, Fax: +98-2634340470, \\ E-mail:M_Hemati1@yahoo.com
}

Received: November 17, 2013; Revised: May 28, 2014; Accepted: June 29, 2014

\begin{abstract}
Background: Resistance toward quaternary ammonium compounds (QACs) is widespread among a diverse range of microorganisms and is facilitated by several mechanisms such as biofilm formation.

Objectives: In this study, the effects of benzalkonium chloride on planktonic growth and biofilm formation by some field isolates of animal bacterial pathogens were investigated.

Materials and Methods: Forty clinical isolates of Escherichia coli, Salmonella serotypes, Staphylococcus aureus and Streptococcus agalactiae (10 isolates of each) were examined for effects of benzalkonium chloride on biofilm formation and planktonic growth using microtiter plates. For all the examined strains in the presence of benzalkonium chloride, biofilm development and planktonic growth were affected at the same concentrations of disinfectant.

Results: The means of strains growth increase after the minimal inhibitory concentration (MIC) were significant in all the bacteria (except for E. coli in 1/32 and S. agalactiae in of 1/8 MIC). Biofilm formation increased with decrease of antiseptics concentration; a significant increase was found in all the samples. The most darkness related to S. aureus and the least to Salmonella.

Conclusions: Bacterial resistance against quaternary ammonium compounds is increasing which can increase the bacterial biofilm formation.
\end{abstract}

Keywords:Biofilms; Benzalkonium Compounds; Salmonella; Staphylococcus; Streptococcus

\section{Background}

Biofilms are known to provide a protective environment for pathogenic bacteria, parasites and viruses, aiding their resistance to antimicrobials, leading to different diseases in animals and humans (1). Bacteria, inside biofilms, have increased resistance to antimicrobial agents (2). The biofilm effect on bacterial resistance is thought to be related to a direct role for the exopolymeric matrix as a diffusion barrier, a chemical reaction of some chemicals with the biofilm matrix, and physiological differences between fixed and suspended organisms (3). Microbial cells in biofilms can easily detach voluntarily or involuntarily from biofilms to aid their dispersal, which represents a very important survival strategy (4). Consequently, bacterial cells which reside in the planktonic phase are thought to be in a phase of moving from one surface to another (5). It is plausible to suggest that these dispersal strategies are therefore the causes of food and water contamination and consequently animal and human infections/ diseases $(6,7)$.

In animal species, the risk of infection is probably considerably greater than the risk in humans. This is due to the difference in animal housing and living environments. In addition to their ability to colonize body surfaces, biofilms are able to colonize artificial surfaces including tubing and implants, such as intravenous catheters, teeth and gingivae, lungs, ears, urogenital tract, and wounds (8).

In the present work, we investigated whether the biofilm formation of some field strains of animal pathogens can be influenced by benzalkonium chloride, at in-use and sub-minimal inhibitory concentrations (sub-MICs). Among cationic antimicrobial agents, quaternary ammonium compounds (QACs) like benzalkonium chlorides have different behaviors. QACs on the contrary of biguanides, fully interact with the membrane and are therefore susceptible to resistance mechanisms mediated through multidrug efflux pumps (9). QACs can cause various degrees of ocular and dermal irritations (10), whereas the toxicity profile with regard to skin irritancy and hypersensitivity of biguanides is excellent at typical in-use levels (11).

Copyright (C) 2015, Ahvaz Jundishapur University of Medical Sciences. This is an open-access article distributed under the terms of the Creative Commons Attribution-NonCommercial 4.0 International License (http://creativecommons.org/licenses/by-nc/4.0/) which permits copy and redistribute the material just in noncommercial usages, provided the original work is properly cited. 


\section{Objectives}

In this study, the effects of benzalkonium chloride on planktonic growth and biofilm formation by some field isolates of animal bacterial pathogens were investigated.

\section{Materials and Methods}

\subsection{Bacterial Strains}

Isolates of Escherichia coli were isolated from dead poultry, referred to the veterinary clinic of Shahrekord University by regional poultry farms. Staphylococcus aureus and Streptococcus agalactiae had been isolated from mastitis cow milk in our previous works $(12,13)$. Salmonella serotypes were isolated from different animals and preserved in the collection of microbiology laboratory of veterinary college. The methods for isolation and identification of all the isolates were based on Queen et al. guidelines (14). Briefly after Gram staining, catalase and oxidase examinations, colonies were pure cultured on sheep blood agar plates (Merck, Darmstadt, Germany).

Hemolysis was scored and then subjected to cyclic adenosine monophosphate (CAMP) (on sheep blood agar), esculin hydrolysis (on TKT [Berlin Treptow-Köpenick]) and rapid hippurate hydrolysis tests for Gram-positive samples. In addition, the growth of isolates on MacConkey agar (Merck, Darmstadt, Germany) was examined. Carbohydrate utilization test was conducted for all the isolates in phenol red broth (BBL) with $1 \%$ final concentration of the following carbohydrates: lactose, maltose, mannitol, raffinose, salicin and trehalose. Positive reactions were indicated by a change from red to yellow after aerobic incubation at $37^{\circ} \mathrm{C}$ for 24 hours.

\subsection{Biofilm Assay}

Biofilm formation was evaluated using end-smooth 96-cell microplates, as explained by Tendolkar and colleagues (13). Briefly, the isolates were grown at $37^{\circ} \mathrm{C}$ in TSB [Tryptic soy broth] (Merck, Darmstadt, Germany). Bacterial cells were then pelleted at $6000 \times \mathrm{g}$ for 10 minutes and the cell pellet was resuspended in $5 \mathrm{~mL}$ of fresh medium. Optical densities (ODs) of bacterial suspensions were measured by spectrophotometer (Jenway, OSA, UK) and normalized to an absorbance of 1.00 at $595 \mathrm{~nm}$. The cultures were diluted 1:40 in fresh tryptone soya broth (TSB) and $200 \mu \mathrm{L}$ of each cell suspension was dispensed into 12 wells in a single row of a sterile 96-well flat-bottom polystyrene microtiter plate. After incubation at $37^{\circ} \mathrm{C}$ for 24 hours, the planktonic cells were aspirated and the wells were washed three times with sterile phosphate-buffered saline (PBS). The plates were inverted and allowed to dry for one hour at room temperature.

For biofilms quantification, $200 \mu \mathrm{L}$ of $0.2 \%$ aqueous crystal violet solution was added to each well and the plates were allowed to stand for 15 minutes. The wells were sub- sequently washed thrice with sterile PBS to wash off the excess crystal violet. The crystal violet bound to the biofilms was extracted with $200 \mu \mathrm{L}$ of an 80:20 (vol/vol) mixture of ethyl alcohol and acetone and the absorbance of the extracted crystal violet was measured at $595 \mathrm{~nm}$. As a control, crystal violet binding to wells was measured, for wells exposed only to the medium with no bacteria. All the biofilm assays were performed in triplicates with 12 replicates for each strain per assay. Interpretation of biofilm production was according to the criteria described by Stepanovic and colleagues (15).

Based on these criteria, the optical density cut-off(ODc) value is defined as: average OD of negative control $+3 \times$ SD (standard deviation) of negative control, and the biofilms producers are categorized as: no biofilm producer, $\leq$ ODc; weak biofilm producer, ODc $<\sim \leq 2 \times$ ODc; moderate biofilm producer, $2 \times$ ODc $<\sim \leq 4 \times$ ODc; strong biofilm producer, $>4 \times$ ODc; where " " stands for average of sample ODs. All the bacterial isolates were examined for biofilm formation and 10 isolates in each genus with strong biofilm productions were selected for determining the MIC of disinfectant on biofilm formation.

\subsection{Disinfectant and Determining the Minimal In- hibitory Concentrations}

The disinfectant used in the present study was benzalkonium chloride (Sigma-Aldrich, Saint Louis, USA). Onepercent solution of benzalkonium chloride was prepared and preserved for further uses. Standard MICs were determined by broth microdilution in three unrelated experiments. Briefly, $50 \mu \mathrm{L}$ of bacterial suspension (containing $2 \times 10^{6} \mathrm{CFU} / \mathrm{mL}$ ) in TSB medium was added to $50 \mu \mathrm{L}$ of serial two-fold dilutions of disinfectant in TSB in microtiter trays. The plates were incubated for 24 hours at $37^{\circ} \mathrm{C}$ and observed for turbidity. The MIC was defined as the lowest concentration of disinfectant, inhibiting visible bacterial growth.

\subsection{Disinfectant Effects Tests on Planktonic Growth and Biofilm Formation}

To test the disinfectant effects on biofilm formation, Houari and Martino methods were used (16). The method briefly includes the following steps: A) Turbidities of fresh culture of the examined bacteria were adjusted to 0.5 McFarland. B) Fifty microliter of the above bacteria and the same amount of different dilutions of disinfectant were mixed (dilutions of 2 MIC, MIC, $1 / 2$ MIC, $1 / 4 \mathrm{MIC}, 1 / 8$ MIC, 1/16 MIC, and 1/32 MIC). C) The microplates were incubated in $37^{\circ} \mathrm{C}$ for 24 hours. D) The absorbance of planktonic growth of bacteria in $630 \mathrm{~nm}$ was determined using ELISA microplate reader (Bio-Tek, Winooski, USA).

\subsection{Statistical Analysis}

The results were analyzed and compared using Duncan multi-range tests in probability level of $5 \%$. 
Ebrahimi A et al.

\section{Results}

\subsection{Properties of Strains}

The most efficient biofilm formations were seen in $S$. aureus and S. agalactiae strains and the least two were observed in E. coli ones. For each strain, the MIC was determined by the conventional two-fold dilution method in TSB, which were $0.047,0.03,0.125$, and $0.125(\mathrm{w} \% \times \mathrm{v} \%)$ for E. coli, S. serotypes, S. aureus and S. agalactiae, respectively. S. aureus and S. agalactiae were the least sensitive strains, and Salmonella was the most sensitive one to benzalkonium chloride.

\subsection{Effects of Benzalkonium Chloride on Bacterial Planktonic and Biofilm Growth}

In Figure 1, biofilm formation and planktonic growth of the four strains in the presence of different concentrations of benzalkonium chloride are presented. The mean of strains growth increased after the MIC reached to the significant level in all bacteria (except for E. coli in 1/32 and $S$. agalactiae in 1/8 concentrations). The rate of the strains growth in the concentration of $1 / 2$ was very negligible and unobservable and growth increase and holes darkness were observable with naked eyes from the MIC of $1 / 4$ and in some of them from $1 / 8$. Statistical diagrams indicated significant increase of planktonic growth in $1 / 8$ MIC and after that. The most planktonic growth was related to S. aureus with an OD of 0.93 and the least darkness was related to Salmonella with an OD of $0.83(\mathrm{P}<0.05)$.

Biofilm formation increased with decrease of antiseptics concentration; a significant increase was found in all the samples. The most darkness was related to $S$. aureus with an OD of 0.49 and the least was related to Salmonella with an OD of 0.36 , which can be a justifier of the high MIC of S. aureus and the low MIC of Salmonella.

\section{Discussion}

The present study was designed to determine the inhibiting effects of benzalkonium chloride on biofilm and planktonic growth of some animal bacterial pathogens. No significant planktonic growth and biofilm formation were observed in the presence of benzalkonium chloride in one and two-fold MICs $(\mathrm{P}<0.05)$. It can imply that benzalkonium chloride concentrations higher than the MIC have similar effects on planktonic growth and biofilm formation and there is no need to use concentrations higher that MIC for controlling bacterial infections. Of course, the rule is gradually voiding in cases including the presence of resistance gens, mutation $(17,18)$, and resistance acquiring $(17,19)$. In addition, organic materials, $\mathrm{pH}$, temperature, water rigidity, chemical harnesses and contact time are involved in the effects of disinfectants (20-22).

The planktonic growth increase from 1/4 MIC to MIC was not concrete and observable (with naked eye), which
Figure 1. The Effect of Benzalkonium Chloride on Planktonic Growth and Biofilm Formation
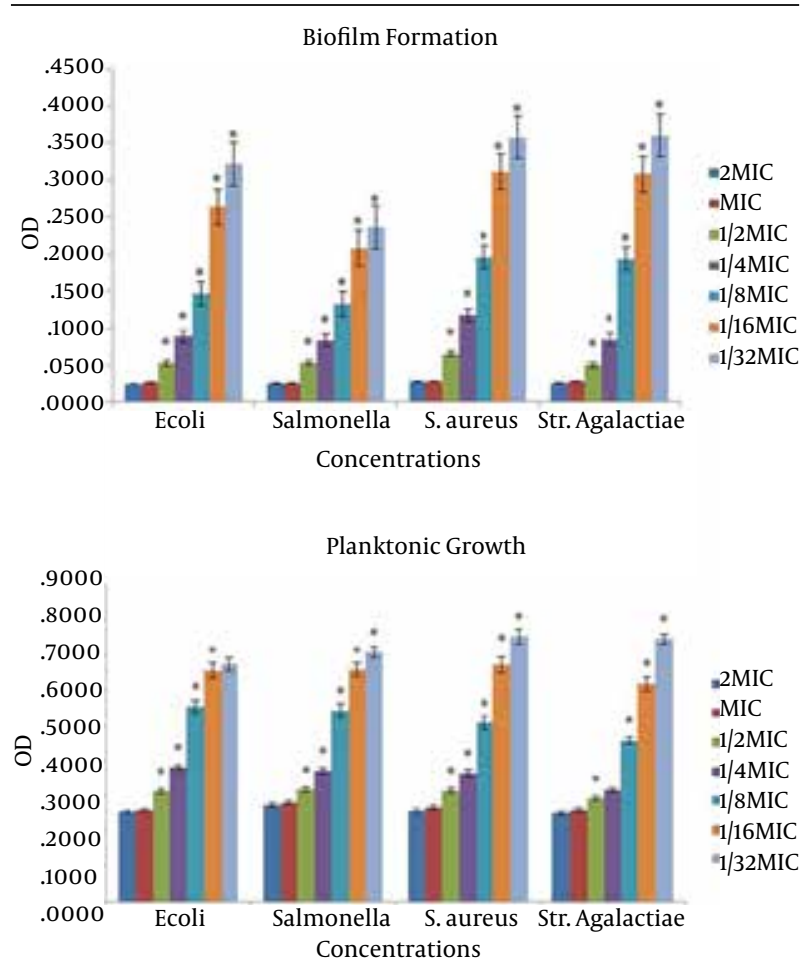

Plates were inoculated with the bacterial suspensions (turbidity of 0.5 McFarland) with benzalkonium chloride at different concentrations and incubated at $37^{\circ} \mathrm{C}$ for 24 hours with an OD of $630 \mathrm{~nm}$. Data are expressed as the mean of three separate experiments in triplicate $( \pm \mathrm{SD})\left({ }^{*} \mathrm{P}<0.05\right)$. Indicates a statistically significant difference from the previous column.

can be a good sign of appropriate effect of benzalkonium chloride on planktonic growth, although as said, limeade OD increase is registered in concentrations less than MIC by ELISA reader. The results were the conclusion of 10 bacterial limeade growth means in different concentrations with triple repetition. However, the biofilm formation increase was concrete after the MIC (because of the release of the absorbed color by the bacteria). More significant increase was found with the MIC decrease.

The above results indicated that bacteria resistance against QACs is increasing and can increase the bacterial biofilm formation. In fact, while these cationic antimicrobial agents at doses below in-use concentrations can be effective to prevent biofilm development of examined bacterial strains, sub-MIC doses of benzalkonium chloride can stimulate the strains biofilm formation. This phenomenon can have deleterious effects, because biofilm formation is thought to play an important role in the survival of virulent strains of food-related and nosocomial staphylococci. Moreover, biofilm formation has been positively correlated with resistance to QACs for staphylococci isolated in the food industry (23). S. aureus 
Ebrahimi A et al.

has been reported as a concern in postoperative wound biofilm infections (24) and mastitis (25). Cross-infection of methicillin-resistant S. aureus (MRSA) between animals and humans has been recognized (26). Akimitsu and colleagues showed that resistant of $S$. aureus to oxacillin and resistance to benzalkonium chloride are closely related (27).

Evidences show that biofilm life manner causes resistance increase against antimicrobial products. Many antibiotherapy treatments, currently used to treat bacterial infections, are aimed at planktonic bacterial cells, as opposed to cells encased in a biofilm; this makes their treatment increasingly problematic. Without adequate diagnostic and treatment protocols to treat veterinary biofilms, their impact will remain a significant challenge. Consequently, one of the bacterial resistance methods is biofilm growth, by which the cells generally survive, because of the disinfectants inability to reach the cells. This will cause bacterial sensitivity reduction (28).

The mode of action of cationic antimicrobials against bacterial cells involves a general perturbation of lipid bilayer membranes (29). Low concentrations of QAC firmly bind to exposed anionic sites on cell membranes. Such interaction can decrease the membrane fluidity and affect osmoregulation and physiological functions of cell membranes (30) as well as biofilm development. Despite valuable properties, concerns have been raised about the widespread and irrational use of disinfectants which could fail the eradication of biofilm-associated microorganisms and serve to select disinfectant-insusceptible microbes among hospital-acquired pathogens $(31,32)$. As a result, the use of benzalkonium chloride as an antimicrobial agent should be avoided and special care should be taken when dealing with inappropriate doses of this antibacterial agent. The suitable concentration of antimicrobials should always be supported by experimental assays. Otherwise, biofilm establishment and bacteria virulence might be favored, contributing to the increased prevalence of surface contamination and biofilm-associated infections.

At higher in-use concentrations, the interactions are more severe and cause the membrane to lose its structural integrity, allowing leakage of cellular materials (8). Stimulation of S. aureus and S. agalactiae biofilm formation by benzalkonium chloride seems to be unrelated to any effect on bacterial growth of planktonic cells; but, effects on cell viability cannot be excluded. Therefore, the presence of a biocide at a low concentration could decrease planktonic viability and select against planktonic growth. A study by Smith and Hunter on the effects of hospital disinfection containing chlorhexidine gluconate $1 \%$, benzalkonium chloride $4 \%$ and triclosan $1 \%$, on the biofilm of multidrug-resistant clinical strains showed that although disinfection may be effective against planktonic populations of bacteria, some disinfectants frequently used in hospitals against growing hospital pathogens as biofilms attached to the surfaces, were inefficient and not able to control the reservoir for nosocomial infections (33).

Recently, there have been some studies reporting biofilm adaptation to the benzalkonium chloride sub-MIC (34-36). I most of the recent studies on disinfection effects of benzalkonium chloride, emphasized on inappropriate effects of the disinfectant on bacterial biofilm formation $(17,19,33)$. Hegstad and colleagues showed that microorganisms' exposure to disinfectants in sub-MIC can lead to the emergence of resistant colonies. The results suggest that although the antimicrobial substances are generally used in very high concentrations, there is always the possibility that some bacteria exposed to sub-MIC concentrations increase their resistance against disinfectants (19).

The results indicated that benzalkonium chloride was able to inhibit biofilm formation of different bacterial species at conventional in-use concentrations. Nevertheless, the biofilm formation induction observed for the strains in the presence of sub-MIC of the disinfectant raises concern over the inappropriate use of cationic disinfectants. Given the prevalence of biofilms in natural environments, it is not surprising that these growth forms are responsible for infection in humans and animals.

\section{Authors' Contributions}

Study concept and design: Majid Hemati and Azizollah Ebrahimi. Statistical analysis and interpretation of data: Hemati. Critical revision of the manuscript for important intellectual content, contribution to the development of the protocol, abstracting the data and preparing the manuscript: Azizollah Ebrahimi, Majid Hemati and Sharareh Lotfalian.

\section{Funding/Support}

This study was supported by the School of Veterinary Sciences, Shahrekord University.

\section{References}

1. Howe AD, Forster S, Morton S, Marshall R, Osborn KS, Wright P, et al. Cryptosporidium oocysts in a water supply associated with a cryptosporidiosis outbreak. Emerg Infect Dis. 2002;8(6):619-24.

2. Gilbert P, Das JR, Jones MV, Allison DG. Assessment of resistance towards biocides following the attachment of micro-organisms to, and growth on, surfaces. JAppl Microbiol. 2001;91(2):248-54.

3. Mah TF, O'Toole GA. Mechanisms of biofilm resistance to antimicrobial agents. Trends Microbiol. 2001;9(1):34-9.

4. Sauer K, Camper AK, Ehrlich GD, Costerton JW, Davies DG. Pseudomonas aeruginosa displays multiple phenotypes during development as a biofilm. J Bacteriol. 2002;184(4):1140-54.

5. Parsek MR, Fuqua C. Biofilms 2003: emerging themes and challenges in studies of surface-associated microbial life. J Bacteriol. 2004;186(14):4427-40.

6. Zottola EA, Sasahara KC. Microbial biofilms in the food process ing industry--should they be a concern? Int J Food Microbiol. 1994;23(2):125-48.

7. Piriou P, Dukan S, Levi Y, Jamge PA. Prevention of bacterial growth in drinking water distribution systems. Water Sci Technol. 1997;35(11-12):283-7.

8. Potera C. Forging a link between biofilms and disease. Science. 1999;283(5409):1837.

9. Lambert PA, Hammond SM. Potassium fluxes, first indications 
of membrane damage in micro-organisms. Biochem Biophys Res Commun. 1973;54(2):796-9.

10. Lin GH, Hemming M. Ocular and dermal irritation studies of some quaternary ammonium compounds. Food Chem Toxicol. 1996;34(2):177-82.

11. Tupker RA, Schuur J, Coenraads PJ. Irritancy of antiseptics tested by repeated open exposures on the human skin, evaluated by non-invasive methods. Contact Dermatitis. 1997;37(5):213-7.

12. Ebrahimi A, Moatamedi A, Lotfalian S, Mirshokraei P. Biofilm formation, hemolysin production and antimicrobial susceptibilities of Streptococcus agalactiae isolated from the mastitis milk of dairy cows in Shahrekord district, Iran. Vet Res Forum. 2013;4(4):269-72.

13. Ebrahimi A, Akhavan Taheri M. Characteristics of staphylococci isolated from clinical and subclinical mastitis cows in Shahrekord, Iran. Iran J Vet Res. 2009;10(3):273-7.

14. Quinn PJ, Carter ME, Markey BK, Carter GR. Clinical veterinary Microbiology. 2nd edLondon: Mosby; 1994.

15. Stepanovic S, Vukovic D, Hola V, Di Bonaventura G, Djukic S, Cirkovic I, et al. Quantification of biofilm in microtiter plates: overview of testing conditions and practical recommendations for assessment of biofilm production by staphylococci. APMIS. 2007;115(8):891-9.

16. Houari A, Di Martino P. Effect of chlorhexidine and benzalkonium chloride on bacterial biofilm formation. Lett Appl Microbiol. 2007;45(6):652-6.

17. Machado I, Lopes SP, Sousa AM, Pereira MO. Adaptive response of single and binary Pseudomonas aeruginosa and Escherichia coli biofilms to benzalkonium chloride. J Basic Microbiol. 2012;52(1):43-52.

18. Pagedar A, Singh J, Batish VK. Adaptation to benzalkonium chloride and ciprofloxacin affects biofilm formation potential, efflux pump and haemolysin activity of Escherichia coli of dairy origin. J Dairy Res. 2012;79(4):383-9.

19. Hegstad K, Langsrud S, Lunestad BT, Scheie AA, Sunde M, Yazdankhah SP. Does the wide use of quaternary ammonium compounds enhance the selection and spread of antimicrobial resistance and thus threaten our health? Microb Drug Resist. 2010;16(2):91-104.

20. Bremer PJ, Monk I, Butler R. Inactivation of Listeria monocytogenes/Flavobacterium spp. biofilms using chlorine: impact of substrate, $\mathrm{pH}$, time and concentration. Lett Appl Microbiol. 2002;35(4):321-5.

21. Cloete TE, Jacobs L, Brozel VS. The chemical control of biofouling in industrial water systems. Biodegradation. 1998;9(1):23-37.

22. Kuda T, Yano T, Kuda MT. Resistances to benzalkonium chloride of bacteria dried with food elements on stainless steel surface. LWT - Food Science and Technology. 2008;41(6):988-93.

23. Moretro T, Hermansen L, Holck AL, Sidhu MS, Rudi K, Langsrud
S. Biofilm formation and the presence of the intercellular adhesion locus ica among staphylococci from food and food processing environments. Appl Environ Microbiol. 2003;69(9):5648-55.

24. Galuppo LD, Pascoe JR, Jang SS, Willits NH, Greenman SL. Evaluation of iodophor skin preparation techniques and factors influencing drainage from ventral midline incisions in horses. J Am Vet Med Assoc. 1999;215(7):963-9.

25. Melchior MB, Fink-Gremmels J, Gaastra W. Comparative assessment of the antimicrobial susceptibility of Staphylococcus aureus isolates from bovine mastitis in biofilm versus planktonic culture. J Vet Med B Infect Dis Vet Public Health. 2006;53(7):326-32.

26. Witte W, Strommenger B, Stanek C, Cuny C. Methicillin-resistant Staphylococcus aureus ST398 in humans and animals, Central Europe. Emerg Infect Dis. 2007;13(2):255-8.

27. A. Kimitsu N, Hamamoto H, Inoue R, Shoji M, Akamine A, Takemori K, et al. Increase in resistance of methicillin-resistant Staphylococcus aureus to beta-lactams caused by mutations conferring resistance to benzalkonium chloride, a disinfectant widely used in hospitals. Antimicrob Agents Chemother. 1999;43(12):3042-3.

28. Campanac C, Pineau L, Payard A, Baziard-Mouysset G, Roques C Interactions between biocide cationic agents and bacterial biofilms. Antimicrob Agents Chemother. 2002;46(5):1469-74.

29. Gilbert P, Moore LE. Cationic antiseptics: diversity of action under a common epithet.J Appl Microbiol. 2005;99(4):703-15.

30. Hugo WB, Longworth AR. The effect of chlorhexidine on the electrophoretic mobility, cytoplasmic constituents, dehydrogenase activity and cell walls of Escherichia coli and Staphylococcus aureus. J Pharm Pharmacol.1966;18(9):569-78.

31. Kawamura-Sato K, Wachino J, Kondo T, Ito H, Arakawa Y. Reduction of disinfectant bactericidal activities in clinically isolated Acinetobacter species in the presence of organic material. J Antimicrob Chemother. 2008;61(3):568-76.

32. Russell AD. Bacterial resistance to disinfectants: present knowledge and future problems. J Hosp Infect. 1999;43 Suppl:S57-68.

33. Smith K, Hunter IS. Efficacy of common hospital biocides with biofilms of multi-drug resistant clinical isolates.J Med Microbiol. 2008;57(Pt 8):966-73.

34. Mangalappalli-Illathu AK, Vidovic S, Korber DR. Differential adaptive response and survival of Salmonella enterica serovar enteritidis planktonic and biofilm cells exposed to benzalkonium chloride. Antimicrob Agents Chemother. 2008;52(10):3669-80.

35. Mangalappalli-Illathu AK, Korber DR. Adaptive resistance and differential protein expression of Salmonella enterica serovar Enteritidis biofilms exposed to benzalkonium chloride. Antimicrob Agents Chemother. 2006;50(11):3588-96.

36. Romanova NA, Wolffs PF, Brovko LY, Griffiths MW. Role of efflux pumps in adaptation and resistance of Listeria monocytogenes to benzalkonium chloride. Appl Environ Microbiol. 2006;72(5):3498-503. 